Proceeding Series of the Brazilian Society of Computational and Applied Mathematics

\title{
Three-dimensional resistive magnetohydrodynamics simulations during high-intensity, long-duration, continuous auroral electrojet activity events and a quiet period: First results
}

\section{Maibys Sierra Lorenzo ${ }^{1}$}

Center for Atmospheric Physics, Meteorology Institute of Cuba Angela León Mecías ${ }^{2}$

Applied Mathematics Department, Faculty of Mathematics and Computer Science, University of Havana, Cuba

Margarete Oliveira Domingues ${ }^{3}$

Laboratório Associado de Computação e Matemática Aplicada (LAC/CTE), Instituto Nacional de Pesquisas Espaciais (INPE)

Odim Mendes, Ezequiel Echer, Varlei Menconi and Paulo R. Jauer ${ }^{4}$

Divisão de Geofísica Espacial DGE/CEA/INPE, São José dos Campos, São Paulo, Brazil

\begin{abstract}
Contributing to the Space Electrodynamics investigations, this work evaluates three kinds of initializations for a resistive three-dimensional magnetohydrodynamics model from the University of Nagoya. One simulation experiment is carried out considering a quiet period, other considering a typical High-Intensity, Long-Duration, Continuous AE activity (HILDCAA) event, and the last one an interplanetary magnetic field $\mathrm{Bz}$, changing according to an idealized event. The data sets for the experiments are created using both smoothed one-minute interplanetary magnetic field components and solar wind plasma parameters extracted from the NASA/GSFC's OMNI data set. As result, reproduction of the earlier work was obtained. Some initial aspects of the simulation has been tested with good performance. New tests and some modifications are being considered in order to improve the code.
\end{abstract}

Keywords. magnetohydrodynamics, HILDCAA, resistive model

\section{Introduction}

Several phenomena originated from the Sun create electrodynamical disturbances in the environment surrounding the Earth. Many efforts have been done by several research

\footnotetext{
${ }^{1}$ maibys.lorenzo@insmet.cu

2angela@matcom.uh.cu

${ }^{3}$ margarete.domingues@inpe.br

${ }^{4}$ odim.mendes@inpe.br, ezequiel.echer@inpe.br,varlei.menconi@inpe.br, pauloricardojauer@gmail.com
} 
groups to unravel features of the High-intensity, long-duration, continuous auroral electrojet activity (HILDCAA) events, as defined by [5]. Independent on the interpretation of them, those occurrences define periods very useful to examine the physical processes of electrodynamical coupling between the solar plasma and the Earth's magnetosphereionosphere system. In general, they do not produce strong effects as other interplanetary causes, but they are stronger than the interplanetary quiet conditions that incide upon the Earth. It is the reason to adopt HILDCAA periods in simulations. Particularly, the response of the near Earth plasma sheet to the variability of the z-component $\left(B_{z}\right)$ of the Interplanetary Magnetic Field (IMF) during HILDCAAs has been studied [5]. This variability is associated with the presence of large-amplitude interplanetary Alfvén waves in the high speed streams that are main cause of HILDCAAs. Results reached by [6], based in the IMP-8 solar wind plasma, magnetic field data and the auroral electrojet index $(A E)$, show that there is a high correlation between the $A E$ and the southward $B_{z}$ component. These results suggest that HILDCAAs are a consequence of enhanced solar wind energy transfer to the magnetosphere caused by magnetic reconnection between the predominantly southward component of the interplanetary Alfvén waves and the magnetic field at the magnetosphere boundary.

Motivated by those kinds of previous results, the present paper aims to study the effects of the variability of the magnetic field behavior during HILDCAA events and to compare the conditions with the conditions during a geomagnetically quiet period. It represents a good opportunity for choice of experimental values to be used in the improvement of a MHD code. The approach used is to initialize a global three dimensional MHD model, based in the code developed by [3], inserting OMNI data sets of interplanetary magnetic field and solar wind plasma parameters. With this study purpose, three simulations are designed for these investigations: one considers a geomagnetically calm period, with no presence of HILDCAAs, other with an ideal HILDCAA described in [1], and a third one with a HILDCAA event. Thus, this article is organized as follows. Section 2 presents the issues of the model. Section 3 the simulation design and the experiment descriptions. Section 4 discusses the results of the simulations. The last section presents the preliminary results of this work.

\section{Model}

The physical-mathematical model is an initial value problem with the MHD and Maxwell's equations written as follows:

$$
\begin{aligned}
\frac{\partial \rho}{\partial t} & =-\nabla \cdot(\mathbf{v} \rho)+D \nabla^{2} \rho, \\
\frac{\partial p}{\partial t} & =-(\mathbf{v} \cdot \nabla) p-\gamma p \nabla \cdot \mathbf{v}+D_{p} \nabla^{2} p, \\
\frac{\partial \mathbf{v}}{\partial t} & =-(\mathbf{v} \cdot \nabla) \mathbf{v}-\frac{1}{\rho} \nabla p+\frac{1}{\rho} \mathbf{J} \times \mathbf{B}+\mathbf{g}+\frac{1}{\rho} \Phi, \\
\frac{\partial \mathbf{B}}{\partial t} & =\nabla \times(\mathbf{v} \times \mathbf{B})+\eta \nabla^{2} \mathbf{B}, \text { and } \\
\mathbf{J} & =\nabla \times\left(\mathbf{B}-\mathbf{B}_{\mathbf{d}}\right),
\end{aligned}
$$


where $\rho$ is the scalar plasma density, $v$ is the flow velocity vector, $p$ the scalar plasma pressure, $B$ is the induction magnetic field vector and $J$ is the current density vector. Details about the other quantities appearing in System 1 can be found in [3].

The normalization quantities in the basic equations are the Earth radius $l_{n}=R_{E}=$ $6.37 \times 10^{6} \mathrm{~m}$, the density of ionosphere $\rho_{l_{n}}=m_{p} n_{l_{n}}\left(n_{l_{n}}=10^{10} \mathrm{~m}^{-3}\right)$, the magnetic field at one earth radius at equator $B_{l_{n}}=3.12 \times 10^{-5} \mathrm{~T}$, the Alfvén velocity at one earth radius $v_{l_{n}}=6.80 \times 10^{6} \mathrm{~m} / \mathrm{s}$, and the Alfvén transit time (normalization time) $t_{l_{n}}=0.937 \mathrm{~s}$. The normalization of the other quantities is derived from the previous ones [3].

\section{$3 \quad$ Numerical aspects}

On this section a brief introduction of the model is presented.

Simulation box For the three study cases selected, a quarter simulation box assuming symmetry conditions is used. Figure 1 shows the Cartesian system used similar to the one in [3]. Earth is located at $(x, y, z)=(0,0,0)$ where $-x_{0} \leq x \leq x_{0},-y_{0} \leq y \leq 0$ and $0 \leq$ $z \leq z_{0}$ with $x_{0}=y_{0}=z_{0}=45 R_{E}$. The mesh dimensions are $(n x, n y, n z)=(180,60,60)$ with a resolution of $\Delta x=\Delta y=\Delta z=0.5 R_{E}$ in all directions.

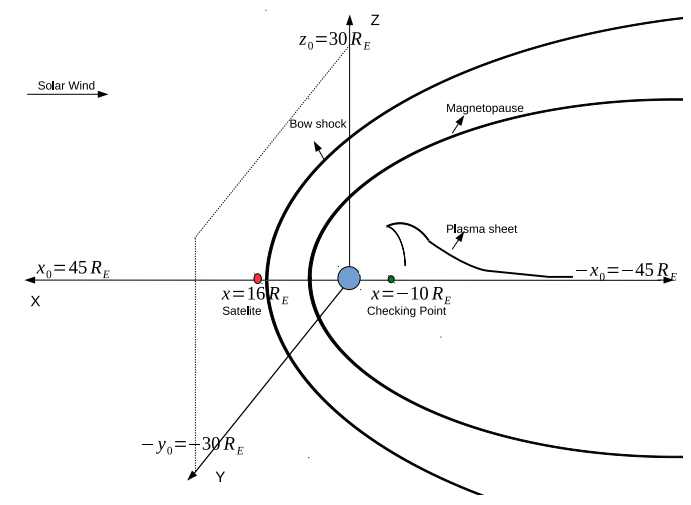

Figure 1: Simulation box using cartesian system in GSM coordinates as proposed by [3]. The blue circle at the center is the Earth, the red circle indicate the satellite position and the green one indicate the point where the behaviour of the physical quantities is studied.

Boundary conditions For the simulation domain, the boundary conditions imposed for each of the physical quantities $\phi(\rho, V, p, B)$ are the same used in [3] but, at the upstream boundary, that is $x=x_{0}$, the 1 minute mean values measured by various spacecraft near the Earth's orbit are used as input for $\left(\rho, V_{x}, P, B_{z}\right)$. These are connected with the quantities computed in the simulation by means of a smoothed function every $32 \Delta t$.

Initial Conditions The initialization is done with a steady state ionosphere in the neighborhood of the Earth [3]. The initial conditions are the following: density $\rho_{0}=\xi^{-3}$ 
for $\rho_{0} \geq 0.2 \rho_{s w}$ and $\rho_{0}=0.2 \rho_{s w}$ for $\rho_{0}<\rho_{s w}$, plasma pressure $p_{0}=\xi^{-2} p_{00}$ for $p_{0} \geq p_{s w}$ and $p_{0}=p_{s w}$ for $p_{0}<p_{s w}$, gravity force $g=\frac{-g_{0}}{\xi^{3}}(x, y, z)$ and dipole magnetic field $B_{d}=\frac{1}{\xi^{5}}\left(-3 x z,-3 y z, x^{2}+y^{2}-2 z^{2}\right)$ where $g_{0}=1.35 \times 10^{-6}$ and $p_{00}=5.4 \times 10^{-7}$. The solar wind parameters and the interplanetary magnetic field $\rho_{s w}, p_{s w}$ and $v_{s w}$, were taken from the OMNI data set.

Finite difference solver A modified leapfrog method introduced by [4] is used. The main modification consists in combining the leapfrog scheme with a two-steps Lax-Wendroff method, also used before in [4]. This combination allows leapfrog method to inherits the stability properties of the two-steps Lax-Wendroff difference scheme. For example, let us consider an equation of the form, $\frac{\partial F}{\partial t}=-\frac{\partial}{\partial x} F_{x}-\frac{\partial}{\partial y} F_{y}-\frac{\partial}{\partial z} F_{z}-F$ For $F_{i, j, k}^{m}=F\left(t_{m}, x_{i}, y_{j}, z_{k}\right)$ and adopting the notation $\Delta x, \Delta y, \Delta z$, as spatial step and $\Delta t$ as time step; $i+1, j+1, k+1$ and $m+1$ are the same as $x_{i}+\Delta x, y_{j}+\Delta y, z_{k}+\Delta z$ and $t_{m}+\Delta t$. Therefore, the application of the modified leapfrog method has a first step as

$$
\begin{aligned}
F_{i+\frac{1}{2}, j+\frac{1}{2}, k+\frac{1}{2}}^{m} & =\frac{1}{8}\left(F_{i, j, k}^{m}+F_{i+1, j, k}^{m}+F_{i, j+1, k}^{m}+F_{i, j, k+1}^{m}+F_{i+1, j+1, k}^{m}+F_{i+1, j, k+1}^{m}+F_{i, j+1, k+1}^{m}+F_{i+1, j+1, k+1}^{m} .\right) \\
F_{i+\frac{1}{2}, j+\frac{1}{2}, k+\frac{1}{2}}^{m+\frac{1}{2}} & =F_{i+\frac{1}{2}, j+\frac{1}{2}, k+\frac{1}{2}}^{m}-\frac{\Delta t}{2} F_{i+\frac{1}{2}, j+\frac{1}{2}, k+\frac{1}{2}}^{m} \\
& -\frac{\Delta t}{8 \Delta x}\left(F_{i+1, j, k}^{m}+F_{i+1, j+1, k}^{m}+F_{i+1, j, k+1}^{m}+F_{i+1, j+1, k+1}^{m}-F_{i, j, k}^{m}-F_{i, j+1, k}^{m}-F_{i, j, k+1}^{m}-F_{i, j+1, k+1}^{m}\right) \\
& -\frac{\Delta t}{8 \Delta y}\left(F_{i, j+1, k}^{m}+F_{i+1, j+1, k}^{m}+F_{i, j+1, k+1}^{m}+F_{i+1, j+1, k+1}^{m}-F_{i, j, k}^{m}-F_{i+1, j, k}^{m}-F_{i, j, k+1}^{m}-F_{i+1, j, k+1}^{m}\right) \\
& -\frac{\Delta t}{8 \Delta z}\left(F_{i, j, k+1}^{m}+F_{i+1, j, k+1}^{m}+F_{i, j+1, k+1}^{m}+F_{i+1, j+1, k+1}^{m}-F_{i, j, k}^{m}-F_{i+1, j, k}^{m}-F_{i, j+1, k}^{m}-F_{i+1, j+1, k}^{m}\right),
\end{aligned}
$$

and a second step as,

$$
\begin{aligned}
F_{i, j, k}^{t+\frac{1}{2}}= & \frac{1}{8}\left(F_{i-\frac{1}{2}, j-\frac{1}{2}, k-\frac{1}{2}}^{t+\frac{1}{2}}+F_{i+\frac{1}{2}, j+\frac{1}{2}, k+\frac{1}{2}}^{t+\frac{1}{2}}+F_{i-\frac{1}{2}, j+\frac{1}{2}, k-\frac{1}{2}}^{t+\frac{1}{2}}+F_{i+\frac{1}{2}, j+\frac{1}{2}, k-\frac{1}{2}}^{t+\frac{1}{2}}\right. \\
& \left.F_{i+\frac{1}{2}, j-\frac{1}{2}, k+\frac{1}{2}}^{t+\frac{1}{2}}+F_{i-\frac{1}{2}, j+\frac{1}{2}, k+\frac{1}{2}}^{t+\frac{1}{2}}+F_{i+\frac{1}{2}, j+\frac{1}{2}, k+\frac{1}{2}}^{t+\frac{1}{2}}\right) \\
F_{i, j, k}^{t+1}= & F_{i, j, k}^{t}-\Delta t F_{i, j, k}^{t+\frac{1}{2}} \\
- & \frac{\Delta t}{4 \Delta x}\left(F_{i+\frac{1}{2}, j-\frac{1}{2}, k-\frac{1}{2}}^{t+\frac{1}{2}}+F_{i+\frac{1}{2}, j+\frac{1}{2}, k-\frac{1}{2}}^{t+\frac{1}{2}}+F_{i+\frac{1}{2}, j-\frac{1}{2}, k+\frac{1}{2}}^{t+\frac{1}{2}}+F_{i+\frac{1}{2}, j+\frac{1}{2}, k+\frac{1}{2}}^{t+\frac{1}{2}}\right. \\
- & \left.F_{i-\frac{1}{2}, j+\frac{1}{2}, k-\frac{1}{2}}^{t+\frac{1}{2}}-F_{i-\frac{1}{2}, j+\frac{1}{2}, k-\frac{1}{2}}^{t+\frac{1}{2}}-F_{i-\frac{1}{2}, j-\frac{1}{2}, k+\frac{1}{2}}^{t+\frac{1}{2}}-F_{i-\frac{1}{2}, j+\frac{1}{2}, k+\frac{1}{2}}^{t+\frac{1}{2}}\right) \\
- & \frac{\Delta t}{4 \Delta y}\left(F_{i-\frac{1}{2}, j+\frac{1}{2}, k-\frac{1}{2}}^{t+\frac{1}{2}}+F_{i+\frac{1}{2}, j+\frac{1}{2}, k-\frac{1}{2}}^{t+\frac{1}{2}}+F_{i-\frac{1}{2}, j+\frac{1}{2}, k+\frac{1}{2}}^{t+\frac{1}{2}}+F_{i+\frac{1}{2}, j+\frac{1}{2}, k+\frac{1}{2}}^{t+\frac{1}{2}}\right. \\
- & \left.F_{i-\frac{1}{2}, j-\frac{1}{2}, k-\frac{1}{2}}^{t+\frac{1}{2}}-F_{i+\frac{1}{2}, j-\frac{1}{2}, k-\frac{1}{2}}^{t+\frac{1}{2}}-F_{i-\frac{1}{2}, j-\frac{1}{2}, k+\frac{1}{2}}^{t+\frac{1}{2}}-F_{i+\frac{1}{2}, j-\frac{1}{2}, k+\frac{1}{2}}^{t+\frac{1}{2}}\right) \\
- & \frac{\Delta t}{4 \Delta z}\left(F_{i-\frac{1}{2}, j-\frac{1}{2}, k+\frac{1}{2}}^{t+\frac{1}{2}}+F_{i+\frac{1}{2}, j-\frac{1}{2}, k+\frac{1}{2}}^{t+\frac{1}{2}}+F_{i-\frac{1}{2}, j+\frac{1}{2}, k+\frac{1}{2}}^{t+\frac{1}{2}}+F_{i+\frac{1}{2}, j+\frac{1}{2}, k+\frac{1}{2}}^{t+\frac{1}{2}}\right. \\
- & \left.F_{i-\frac{1}{2}, j-\frac{1}{2}, k-\frac{1}{2}}^{t+\frac{1}{2}}-F_{i+\frac{1}{2}, j-\frac{1}{2}, k-\frac{1}{2}}^{t+\frac{1}{2}}-F_{i-\frac{1}{2}, j+\frac{1}{2}, k-\frac{1}{2}}^{t+\frac{1}{2}}-F_{i+\frac{1}{2}, j+\frac{1}{2}, k-\frac{1}{2}}^{t+\frac{1}{2}}\right)
\end{aligned}
$$

This difference scheme needs $F_{i, j, k}^{m}$ in $2 \leq i \leq n x+1,2 \leq j \leq n y+1,2 \leq k \leq n z+1$ for $m=0$. According to the stability condition of the difference scheme, $V_{A}^{\max } \Delta t<(1 / 2) \Delta x$, where $V_{A}^{\max }$ is the maximum Alfvén velocity in the computational domain, the time step 
used is $\Delta t=0.25 \mathrm{~s}$. Also this method computes a temporally forward difference in the first step and change to a central difference in the second step. It depends on the time step selected for the Eq. 2 and 3. With the use of the term $F_{i, j, k}^{t+\frac{1}{2}}$ in Eq. 2, we can obtain the two-step Lax-Wendroff method or the leapfrog method. Thus, the modification proposed in [4] indicates that in the first step of integration the Lax-Wendroff method is used, and in the next time steps the leapfrog method is used. The idea behind this combination is allows a balance between numerical dispersion and diffusion.

Experiments Numerical simulations are conducted for three different situations: idealized HILDCAA event, a quiet event, and a HILDCAA event. For the first case a $B z$ periodic square wave with 40 minutes of periodicity is used as a driver, following the ideas presented in [1]. To build the initial condition, the value of the solar wind is $v_{s w}=\left(v_{x}, 0,0\right)$ with $v_{x}=300 \mathrm{~km} / \mathrm{s}$, the pressure $p=2.8 \times 10^{-8} \mathrm{~N} / \mathrm{m}^{2}$, and $\rho=5 \times 10^{-4} \mathrm{~cm}^{-3}$. The simulations conducted with the satellite data correspond to July $18^{\text {th }}$ of 2006 for the quiet period, and April $20^{\text {th }}$ of 2003 for the HILDCAA event.

We used the plasma and the magnetic field data sets with a duration of 600 minutes. These time series of real data are smoothed using a Hanning window with 120 points, initially for test, in all variables to improve the stability of the code and avoid a blow up of the runs. It has obtained success, but some ideas have been discussed in order to improve the real conditions for the simulation.

Figure 2(a) shows the geomagnetic quiet period and the smoothed version used in the initialization of the model. On one hand, we can observe the low values of the $A E$ index, below of $200 \mathrm{nT}$, and also low values of all the plasma parameters and the interplanetary magnetic field components compared to the HILDCAA event. On the other hand, Figure 2(b) the HILDCAA event has an intense activity in the $A E$ index and the components of magnetic field show fluctuations due to the presence of Alfvén waves [2].
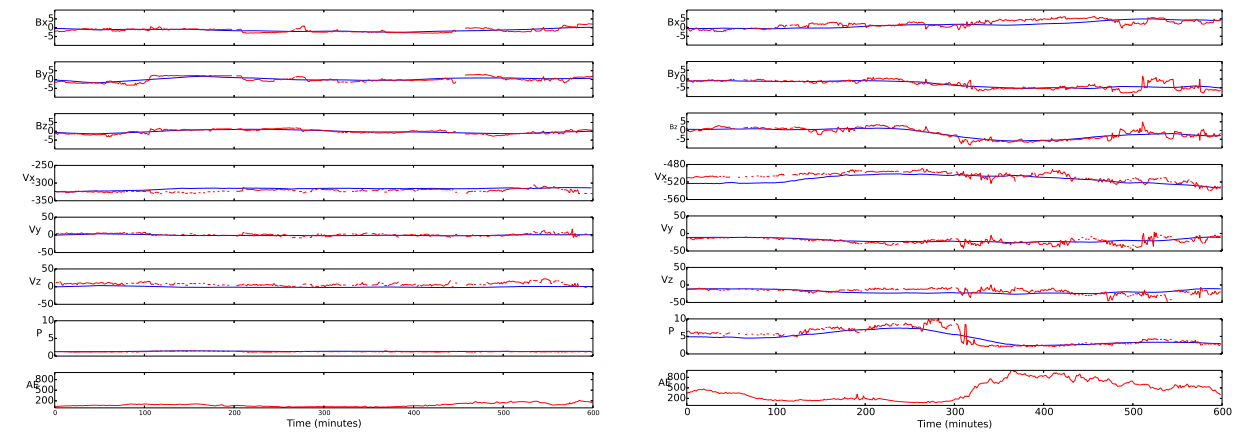

Figure 2: Solar wind parameters and IMF for the study cases, red lines are the real data and the blue lines are the smoothed version of real data using a Hanning window. quiet period (a), and HILDCAA event (b). 


\section{Numerical Results}

Figure 3 presents the results of the idealized and real cases. It shows the time variation of $B z$ component of the interplanetary magnetic field, pressure $p$, density $\rho$ and the speed $v_{x}$. These simulated time series are taken in the magnetospheric tail at $x=-10 R_{E}$. In Figure 3(a), the response of each quantity exhibits a periodic behaviour approximately with 20 minutes of delay in relation with the $B z$ component of IMF (an acceptable value). Notice that the pressure and density reaches the maximum value at the end of northwards period of $B z$. The velocity initially presents oscillations more delayed, and after 500 minutes start to show an equal oscillating behaviour. In general this is consistent with the results achieved by [1] where their found a delay of 15 minutes. However, in order to explore better the physical reasons, some study conditions have been prepared for future tests.

Observing the time variation of the simulated quantities for the quiet period case, one can see that the magnetospheric response is smooth with no abrupt fluctuations (Figure $3(\mathrm{~b})$ ). However, for values related to the real HILDCAA case (Figure 3(c)), the physical quantities present strong variations after 300 minutes of simulation, more pronounced in the density. Figure 4 allows a comparison between the idealized event and the real one at simulation time $t=100$ minutes. In the bow shock region, the pressure has very strong oscillations in the simulation with real data (Figure 4(b)). In relation with the idealized case, the values of the pressure are two orders higher.
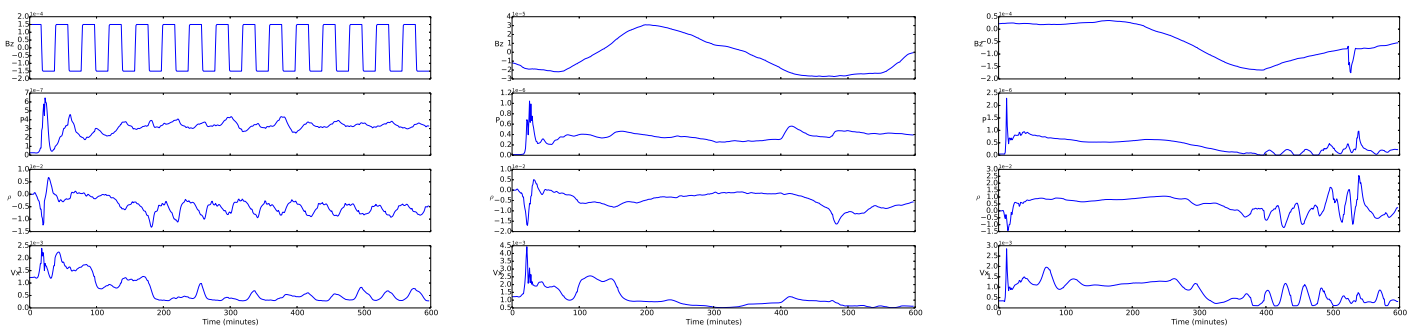

Figure 3: Time variation of pressure $p$, density $\rho$ and convection speed $v_{x}$ in the near Earth plasma sheet for $x=-10 R_{E}$, according to the periodic function of IMF $B z(\mathrm{a})$ and data from the OMNI data set inserted for quiet period simulation (b), and HILDCAA event simulation(c)
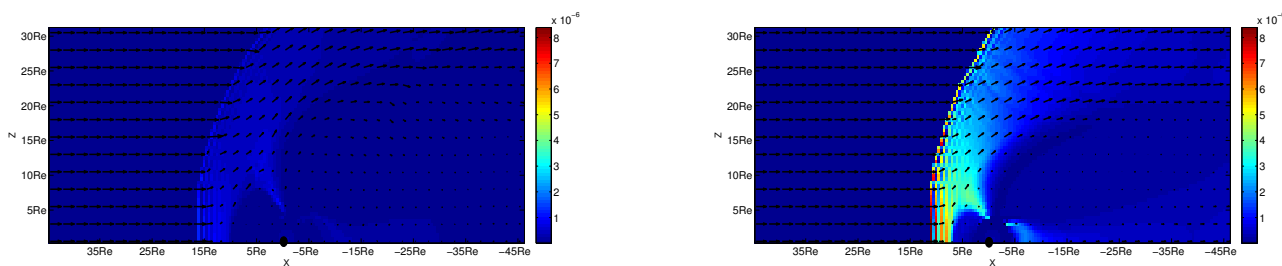

Figure 4: Flow velocity $\mathbf{v}$ and a contour of the pressure $p$ for the noon-midnight meridian plane $(y=0)$ for $t=100 \mathrm{~min}$. Idealized HILDCAA event (a), and HILDCAA event (b). 


\section{Final remarks}

Roughly speaking the achieved simulation results are a first step in the recreation and understanding of HILDCAA large scale scenario. There are still many steps to be improved in the initialization, in the extraction of dynamics, and in the simulation itself. However, the results presented here indicate that interplanetary parameter conditions during HILDCAA events can contribute with large disturbance, which is consistent with the idealized one. Moreover, the results confirm that a scenario different from quiet conditions has been developed, which allows to use this code as a tool to study HILDCAA events. Further work will be done to improve these pitfalls and we expect to study other configurations to contribute to the HILDCAA events understanding.

\section{Acknowledgements}

The authors thank financial support from CNPq-TWAS, FINEP, LAC/INPE and DGE/INPE for the computational facilities, and OMNI team for the data sets. Also authors thanks Prof. Ogino from U. of Nagoya for the 3D-MHD code and discussions that motivated this work.

\section{References}

[1] W.D. Gonzalez, F.L. Guarnieri, A.L. Clua-Gonzalez, E. Echer, M.V. Alves, T. Ogino and B.T. Tsurutani, Magnetospheric Energetics During HILDCAAs, Recurrent Magnetic Storms: Corotating Solar Wind Streams Geophysical Monograph Series 167, (2006).

[2] F.L. Guarnieri, TB.T. Tsurutani, W.D. Gonzalez, A.L. Clua-Gonzalez, M. Grande, F. Soraas and E. Echer, Icme and cir storms with particular emphases on hildcaa events, ILWS WORKSHOP (2006).

[3] T. Ogino, A three dimensional MHD simulation of the interaction of the solar wind with the Earth's magnetosphere, The generation of field aligned currents, J. Geophys. Res., 91, 6791, (1986).

[4] T. Ogino, R. J. Walker and M. Ashour-Abdalla, A global magnetohydrodynamics of the magnetostheath and magnetosphere when the interplanetary magnetic field is northward, IEEE, Trans. Plasma Sci., 20(6),817, (1992).

[5] B.T. Tsurutani and W.D. Gonzalez, The cause of High-Intensity, Long-Duration, Continuous AE activity (HILDCAAs), interplanetary Alfvén wave trains. Planetary and Space Science, 35, 405, (1987).

[6] B.T. Tsurutani, T. Gould, B.E. Goldstein, W.D. Gonzalez and M. Sugiura, Interplanetary Alfvén waves and auroral (substorm) activity, IMP-8, J. Geophys. Res., 95, 2241, (1990). 